\title{
Cofilin-1 is an essential redox sensor for NLRP3 inflammasome activation
}

\author{
YH Park, D Kastner, JJ Chae \\ From 8th International Congress of Familial Mediterranean Fever and Systemic Autoinflammatory Diseases \\ Dresden, Germany. 30 September - 3 October 2015
}

\section{Introduction}

NLRP3 has a pivotal role in nucleating the inflammasome, a cytoplasmic multiprotein complex that mediates the maturation of the proinflammatory cytokine interleukin$1 \beta$ (IL-1 $\beta$ ) by activating caspase- 1 . Mutations in the gene encoding NLRP3 cause a spectrum of autoinflammatory disease, the cryopyrin-associated periodic syndromes (CAPS). It has been reported that generation of reactive oxygen species (ROS) is a major NLRP3 inflammasomeactivating factor. However, the molecular mechanism by which a change in cellular redox state leads to NLRP3 inflammasome activation has not been elucidated. Here we show that cofilin-1, a redox sensitive actin binding protein, is involved in NLRP3 inflammasome activation.

\section{Objectives}

To investigate how ROS activates the NLRP3 inflammasome.

\section{Methods}

Cell culture supernatants from bone marrow derived macrophages (BMDMs) of wild-type or NLRP3-KO mice were analyzed by mass spectrometry. Inflammasome activation was analyzed by Western blotting of secreted IL$1 \beta$ or ASC oligomerization. The interaction of NLRP3 with cofilin-1 was examined by co-immunoprecipitation from BMDMs or transfected cells.

\section{Results}

Cofilin-1 is highly secreted along with IL- $1 \beta$ from LPSprimed BMDMs in response to the known NLRP3 activator, ATP, whereas knockdown of cofilin-1 reduces NLRP3 inflammasome activation. Cofilin-1 directly interacts to the nucleotide-binding domain (NBD) of the NLRP3 protein in LPS-primed BMDMs. However, cofilin-1 is dissociated

$\mathrm{NIH/NHGRI}$, Bethesda, MD, USA 